\title{
Gastrointestinal bleeding rates in recipients of nonpulsatile and pulsatile left ventricular assist devices
}

\author{
Sheri Crow, MD, MS, ${ }^{\mathrm{a}}$ Ranjit John, MD, ${ }^{\mathrm{b}}$ Andrew Boyle, MD, ${ }^{\mathrm{c}}$ Sara Shumway, MD, ${ }^{\mathrm{b}}$ Kenneth Liao, MD, PhD, \\ Monica Colvin-Adams, MD, ${ }^{\mathrm{c}}$ Carol Toninato, RN, ${ }^{\mathrm{b}}$ Emil Missov, MD, ${ }^{\mathrm{c}}$ Marc Pritzker, MD, ${ }^{\mathrm{c}}$ Cindy Martin, MD, ${ }^{\mathrm{c}}$ \\ Daniel Garry, MD, PhD, ${ }^{\mathrm{c}}$ William Thomas, $\mathrm{PhD},{ }^{\mathrm{d}}$ and Lyle Joyce, $\mathrm{MD}, \mathrm{PhD}^{\mathrm{b}}$
}

\begin{abstract}
Objective: Pulsatile and nonpulsatile left ventricular assist devices are effective in managing congestive heart failure. Despite early evidence for clinical efficacy, the long-term impact of nonpulsatile flow on end-organ function remains to be determined. Our goal was to compare rates of gastrointestinal bleeding in nonpulsatile and pulsatile device recipients.
\end{abstract}

\begin{abstract}
Methods: In a retrospective review of 101 left ventricular assist device recipients (55 nonpulsatile, 46 pulsatile) from October 31,2003, to June 1, 2007, at a single center, gastrointestinal bleeding was defined as guaiac-positive stool with hemoglobin drop requiring transfusion of at least 2 units of packed red blood cells. To assess bleeding risk outside the initial postoperative course, any patients with a device in place for 15 days or less was excluded.

Results: Twelve nonpulsatile and 3 pulsatile left ventricular assist device recipients had gastrointestinal bleeding 16 days or longer after device implantation. The event rates were 63 events/100 patient-years for nonpulsatile devices and 6.8 events/100 patient-years for pulsatile devices $(P=.0004)$. This difference persisted for bleeding occurring 31 days or longer after device implantation, with 46.5 events $/ 100$ patient-years for nonpulsatile devices versus 4.7 events $/ 100$ patient-years for pulsatile devices $(P=.0028)$. Mortalities were similar between groups ( $15 \%$ nonpulsatile vs $17 \%$ pulsatile, $P=.6965)$.

Conclusion: Patients with nonpulsatile left ventricular assist devices appear to have a higher rate of gastrointestinal bleeding events than do pulsatile left ventricular assist device recipients. Further prospective evaluation is needed to determine potential etiologies and strategies for reducing gastrointestinal bleeding in this population.
\end{abstract}

Five million Americans carry the diagnosis of congestive heart failure (CHF). ${ }^{1}$ The large discrepancy between donor organ supply and demand has necessitated the development of alternative methods for treating cardiac failure. In addition, a growing number of people need cardiac support but are ineligible for heart transplantation. The clinical success of ventricular assist devices offers an innovative strategy for increasing the life expectancy of patients with CHF. In the Randomized Evaluation of Mechanical Assistance for the Treatment of Congestive Heart Failure (REMATCH) trial, ${ }^{2}$ left ventricular assist device (LVAD) recipients had 1- and 2-year survivals 2 times greater than those of patients treated with traditional medical therapy alone. This landmark trial established the LVAD as a successful alternative to medical therapy for patients not eligible for heart transplantation. ${ }^{2}$ Additional studies have demonstrated similar efficacy for LVAD use as a bridge

From the Division of Pediatric Critical Care Medicine, Mayo Clinic, Rochester, Minn, ${ }^{\text {a }}$ and the Divisions of Cardiothoracic Surgery, ${ }^{\mathrm{b}}$ Cardiology, ${ }^{\mathrm{c}}$ and Biostatistics, Department of Medicine, University of Minnesota, Minneapolis, Minn.

Received for publication April 21, 2008; revisions received June 17, 2008; accepted for publication July 6, 2008 .

Address for reprints: Sheri Crow, MD, MS, 200 First St SW, Rochester, MN 55905 (E-mail: crow.sheri@mayo.edu).

J Thorac Cardiovasc Surg 2009;137:208-15

$0022-5223 / \$ 36.00$

Copyright (c) 2009 by The American Association for Thoracic Surgery doi:10.1016/j.jtcvs.2008.07.032 to transplant (BTT) in patients awaiting donor heart availability. ${ }^{3}$

Now that clinical efficacy has been confirmed, we must direct our focus toward identification of the best device mechanism for delivering mechanical support. The LVADs being used in clinical practice can be divided into two categories according to the type of flow they provide: pulsatile and nonpulsatile. The earliest Food and Drug Administration (FDA)-approved devices provide pulsatile flow mimicking the native cardiac physiology. Unfortunately, features of the pulsatile design often limit device durability, necessitating replacement within 15 to 18 months. In addition, size and weight limit this device type's use in children and small adults. The newer, more compact nonpulsatile devices provide improved ease of implantation and have the potential for improved durability. Preliminary data suggest that pulsatile and nonpulsatile devices have comparable outcomes and equivalent risk profiles. ${ }^{4}$ Early follow-up of nonpulsatile device recipients demonstrate improved functional status and quality of life at 3 months, with a 6-month survival of $75 \% .^{5}$ As survival improves, long-term follow-up of these patients becomes essential to demonstrate efficacy and safety beyond the first postoperative year.

The impact of nonpulsatile flow on end-organ function over time remains unknown. A 6-month follow-up of 10 patients implanted with the Jarvik 2000 device (Jarvik Heart, Inc, New York, NY) demonstrated not only preservation 


\begin{tabular}{|c|c|}
\hline \multicolumn{2}{|c|}{ Abbreviations and Acronyms } \\
\hline AS & $=$ aortic stenosis \\
\hline AVM & $=$ arteriovenous malformation \\
\hline BMI & $=$ body mass index \\
\hline BTT & $=$ bridge to transplant \\
\hline $\mathrm{CHF}$ & $=$ congestive heart failure \\
\hline DT & $=$ destination therapy \\
\hline FDA & $=$ Food and Drug Administration \\
\hline HMW & $=$ high - molecular weight \\
\hline INR & $=$ international normalized ratio \\
\hline LVAD & $=$ left ventricular assist device \\
\hline REMA & $\begin{aligned} & \text { Randomized Evaluation of } \\
& \text { Mechanical Assistance for the } \\
& \text { Treatment of Congestive Heart } \\
& \text { Failure }\end{aligned}$ \\
\hline vWF & $=$ von Willebrand factor \\
\hline
\end{tabular}

of but also improvement in hepatic and renal function. ${ }^{6}$ Some end organs, however, may be more sensitive to nonpulsatile flow. Early case reports have suggested that gastrointestinal bleeding may be a more frequent complication with nonpulsatile devices. Letsou and colleagues ${ }^{7}$ reported that of 21 patients who received a nonpulsatile LVAD, 3 had a gastrointestinal bleeding event. ${ }^{7}$ We sought to investigate this potential complication by comparing the incidence of gastrointestinal bleeding between patients implanted with nonpulsatile and pulsatile LVADs at a single center during the same time period.

\section{MATERIALS AND METHODS Patients}

After obtaining approval from the institutional review board, we retrospectively reviewed the cases of all patients with LVADs implanted from October 31, 2003, to June 1, 2007, at the University of Minnesota. The start of the study inclusion period coincides with the placement of the first nonpulsatile device at our center. There were 109 patients who underwent ventricular assist device placement ( 56 nonpulsatile and 53 pulsatile) during this period. We excluded 2 patients who received right ventricular assist devices. In an attempt to assess bleeding risk outside the initial postoperative course, we excluded any patient with a device in place for 15 days or less (1 nonpulsatile and 5 pulsatile), leaving 101 patients for data analysis. None of the 6 patients with an implant duration of 15 days or less had any gastrointestinal bleeding events. Ten patients underwent multiple LVAD placements. For these patients, only the time after the first LVAD implant was included for data analysis.

\section{Device Placement}

LVAD candidates include all patients who are in New York Heart Association functional class IV CHF and have potential for reversible secondary end-organ failure. The eligible patients in this study were classified as receiving the LVAD as BTT or destination therapy (DT). BTT signifies eligibility for cardiac transplantation with rapid deterioration or ongoing heart failure necessitating mechanical support to sustain life. Patients who do not qualify for transplant may have the option of LVAD placement as DT to extend longevity. LVADs implanted for both BTT and DT may be either pulsatile or nonpulsatile devices, depending on a number of factors. All patients who receive nonpulsatile devices are enrolled in one of the ongoing FDA investigational device exemption protocols. The inclusion criteria for the BTT and DT investigational device exemption trials are listed in the Appendix. Patients not meeting inclusion criteria receive an FDA-approved pulsatile device (HeartMate XVE; Thoratec Corporation, Pleasanton, Calif). Any patient, regardless of therapy classification as BTT or DT, with rapid cardiac failure necessitating temporary placement of a Levitronix (Levitronix LLC, Waltham, Mass) LVAD received a pulsatile device once their clinical condition had stabilized. There were 13 patients in our cohort in whom pulsatile LVAD placement was preceded by a Levitronix LVAD.

\section{Anticoagulation}

Nonpulsatile device recipients are placed on a regimen of warfarin sodium and aspirin, with a goal of an international normalized ratio (INR) of 1.5 to 3 . The protocol for pulsatile device recipients is daily aspirin.

\section{Peptic Ulcer Prophylaxis}

The clinical practice at our institution is to use an intravenous protonpump inhibitor while the patient is hospitalized, with transition to oral proton-pump inhibitor therapy in preparation for discharge. The proton-pump inhibitor is continued indefinitely in the outpatient setting.

\section{Data Collection}

Data were collected from the University of Minnesota ventricular assist device database and patient medical records. Preoperative variables included the following: age, sex, diabetes status, body mass index (BMI), sodium concentration, albumin concentration, and etiology of heart failure. Bypass time and device brand were recorded for each patient. Bypass time included the time required to implant the ventricular assist device and to complete any necessary cardiac repairs. The outpatient follow-up data were collected by recording vital signs and laboratory values documented from two subsequent cardiology visits at least 1 month apart. These two values were averaged for each patient. Specific follow-up data collected were as follows: heart rate, systolic and diastolic blood pressures, medication profile, sodium, creatinine, albumin, liver function tests, INR, and activated partial thromboplastin time. When a gastrointestinal bleeding event occurred, the data were averaged from the two clinic visits preceding the gastrointestinal bleeding date.

The development of a gastrointestinal bleeding event was the primary end point for this study. Gastrointestinal bleeding was defined as a guaiac-positive stool and a hemoglobin drop requiring transfusion of at least 2 units of packed red blood cells. INR and activated partial thromboplastin time were documented at presentation, before transfusion. Gastrointestinal bleeding events were classified according to time from device implantation as early (16-30 days after implant) or late (31 days or later). For the 3 patients who had multiple gastrointestinal bleeding events, only the first event was included for data analysis.

\section{Statistical Analysis}

Data for all continuous variables (age, mean arterial pressure, pulse width, laboratory values, implant duration, and bypass time) are presented as mean $\pm \mathrm{SE}$. The Student $t$ test was used to test for statistically significant differences between groups. Binary variables (sex, diabetes status, and device purpose) are presented as totals with percentages and were compared with a $\chi^{2}$ statistic. The primary outcome, gastrointestinal bleeding, was calculated as a bleeding rate of events per 100 patient-years with LVAD and modeled with Poisson regression by the Genmod procedure (SAS version 9.1; SAS Institute, Inc, Cary, NC). Kaplan-Meier survival curves were used to demonstrate bleeding rates with time. BMI was converted to a categoric variable, either greater than $29 \mathrm{~kg} / \mathrm{m}^{2}$ or less than or equal to $29 \mathrm{~kg} / \mathrm{m}^{2}$. We had full access to the data and take responsibility for its integrity. All of us have read and agree to the article as written. 
TABLE 1. Baseline characteristics

\begin{tabular}{|c|c|c|c|}
\hline & Nonpulsatile $(\mathbf{N}=\mathbf{5 5})$ & Pulsatile $(\mathrm{N}=46)$ & $P$ value \\
\hline Age $(y$, mean $\pm S D)$ & $55.1 \pm 1.9$ & $55.7 \pm 1.8$ & .8433 \\
\hline Sex (male) & $42(76 \%)$ & $39(85 \%)$ & .2903 \\
\hline Diabetes (No.) & $18(35 \%, \mathrm{n}=51)$ & $24(53 \%, \mathrm{n}=45)$ & .0754 \\
\hline Preoperative body mass index $\left(\mathrm{kg} / \mathrm{m}^{2}\right.$, mean $\left.\pm \mathrm{SD}\right)$ & $27.1 \pm 0.8$ & $30.9 \pm 1.0(\mathrm{n}=45)$ & .0040 \\
\hline Preoperative sodium $(\mathrm{mEq} / \mathrm{L}$, mean $\pm \mathrm{SD})$ & $136.0 \pm 0.9(\mathrm{n}=50)$ & $137.0 \pm 0.7(\mathrm{n}=40)$ & .3858 \\
\hline Preoperative albumin $(\mathrm{g} / \mathrm{dL}$, mean $\pm \mathrm{SD})$ & $3.6 \pm 0.1(\mathrm{n}=50)$ & $3.3 \pm 0.1(\mathrm{n}=34)$ & .0445 \\
\hline Duration of support $(\mathrm{d}$, mean $\pm \mathrm{SD})$ & $200.4 \pm 21.5$ & $302.7 \pm 27.5$ & .0043 \\
\hline \multicolumn{4}{|l|}{ Device purpose (No.) } \\
\hline Bridge to transplant & $46(84 \%)$ & $38(83 \%)$ & .8907 \\
\hline Destination therapy & $9(16 \%)$ & $8(17 \%)$ & .8907 \\
\hline Cardiopulmonary bypass time (min, mean $\pm \mathrm{SD}$ ) & $124.1 \pm 5.3$ & $149.0 \pm 8.1$ & .0115 \\
\hline Device type (No.) & & & $<.0001$ \\
\hline HeartMate II* & $38(69 \%)$ & & \\
\hline MicroMed $\dagger$ & $8(15 \%)$ & & \\
\hline VentrAssist $\ddagger$ & $9(16 \%)$ & & \\
\hline HeartMate XVE* & & $46(100 \%)$ & \\
\hline Deaths (No.) & $8(15 \%)$ & $8(17 \%)$ & .6965 \\
\hline
\end{tabular}

*Thoratec Corporation, Pleasanton, Calif. †MicroMed Cardiovascular, Inc, Houston, Tex. $\ddagger$ Ventracor Inc, Foster City Calif.

\section{RESULTS \\ Patients}

A total of 101 patients were available for study inclusion and data analysis after exclusion of patients with right ventricular assist devices or a device implant duration of 15 days or less. Of these 101 patients, 55 received nonpulsatile LVADs and 46 received pulsatile LVADs. The baseline characteristics for the nonpulsatile and pulsatile device recipients are outlined in Table 1. Preoperative screening revealed no difference in the gastrointestinal bleeding history between nonpulsatile and pulsatile LVAD recipients. Device purpose, diabetes status, age, sex, and preoperative sodium level did not differ significantly between groups. There was no difference in mortality $(15 \%$ for nonpulsatile vs $17 \%$ for pulsatile, $P=.6965$ ). The pulsatile group had a slightly lower preoperative albumin level $(3.3 \pm 0.1 \mathrm{vs}$ $3.6 \pm 0.1 \mathrm{~g} / \mathrm{dL}$ ), a difference that did reach significance $(P=.0445)$. The nonpulsatile group had a significantly lower preoperative BMI $\left(27.1 \pm 0.8\right.$ vs $30.9 \pm 1.0 \mathrm{~kg} / \mathrm{m}^{2}$, $P=.0040)$ and a shorter bypass duration $(124.1 \pm 5.3 \mathrm{vs}$ $149.0 \pm 8.1$ minutes $P=.0115)$. The pulsatile group had a significantly longer implant duration $(200.4 \pm 21.5$ vs $302.7 \pm 27.5$ days, $P=.0043$ ).

\section{Primary Outcome}

The primary outcome of interest was the rate of gastrointestinal bleeding in nonpulsatile versus pulsatile device recipients. A gastrointestinal bleeding event rate was calculated with the duration of LVAD support as the time at risk (denominator). This rate comparison was calculated three ways, as shown in Table 2. All bleeding events represents every gastrointestinal bleeding event that occurred 16 days or later after implant and includes multiple bleeding events in the same patient. Two patients in this group had 3 gastrointestinal bleeding events each, and 1 patient had 4 bleeding events. The rate for all gastrointestinal bleeding events was nearly 10 times higher in the nonpulsatile group. Time at risk for early and late gastrointestinal bleeding rates begins with device implantation and ends with the first gastrointestinal bleeding event occurring 15 (early) or 31 (late) days after implantation. The nonpulsatile early and late gastrointestinal bleeding rates were 7 and 10 times higher, respectively, than were pulsatile bleeding rates (Table 2). There were 15 first-time gastrointestinal bleeding events after 15 days, 12 in the nonpulsatile group and 3 in the pulsatile group, $\left(P=.0313, \chi^{2}\right.$ test $)$.

The time to first gastrointestinal bleeding event after 15 days is represented in the Kaplan-Meier curve shown in Figure 1 . The log-rank $\chi^{2}$ test statistic for homogeneity across the device type strata is 7.4565 , with a $P$ value of .0063 .

\section{Postimplant Comparisons}

The postimplant characteristics of patients receiving the two device types are summarized in Table 3. Creatinine,

TABLE 2. Gastrointestinal bleeding event rates

\begin{tabular}{cccc}
\hline Implant duration & $\begin{array}{c}\text { Nonpulsatile } \\
(\mathbf{n}=\mathbf{5 5})\end{array}$ & $\begin{array}{c}\text { Pulsatile } \\
(\mathbf{n}=\mathbf{4 6})\end{array}$ & $\boldsymbol{P}$ value \\
\hline $\begin{array}{l}\text { First bleeding event after } 15 \mathrm{~d} \\
\quad \text { Events/100 patient-y }\end{array}$ & 47.7 & 7.3 & .0036 \\
$\quad$ Total & 12 & 3 & .0013 \\
First bleeding event after 30 d & & & \\
$\quad$ Events/100 patient-y & 46.5 & 4.7 & .0028 \\
$\quad$ Total & 12 & 2 & .0114 \\
All bleeding events after 15 d & 63 & 6.8 & .0004 \\
$\quad$ (events/100 patient-y) & & & \\
\hline
\end{tabular}




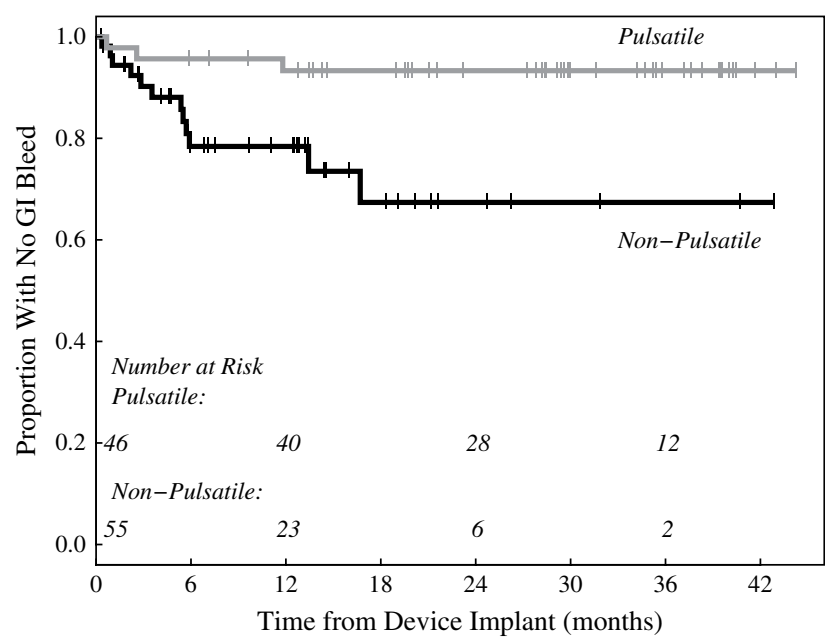

FIGURE 1. Gastrointestinal (GI) bleeding events with time after left ventricular assist device placement in 101 patients.

sodium, albumin, liver function tests (aspartate aminotransferase, alanine aminotransferase, total bilirubin), and activated partial thromboplastin time were similar between groups. As expected in the post implantation comparisons, heart rate (nonpulsatile $84.2 \pm 1.6$ beats/min vs pulsatile 68.2 \pm 1.6 beats $/ \mathrm{min}$ ) and pulse width (nonpulsatile $32.2 \pm 1.5 \mathrm{vs}$ pulsatile $59.0 \pm 2.6)$ were significantly different $(P<.0001$ for both), but mean arterial pressure was not (nonpulsatile $81.1 \pm 1.8 \mathrm{~mm} \mathrm{Hg}$ vs pulsatile $84.8 \pm 1.9 \mathrm{~mm} \mathrm{Hg}$, $P=.1727)$. Anticoagulation with warfarin sodium in the nonpulsatile group resulted in a significant difference in INR (nonpulsatile $2.0 \pm 0.1$ vs pulsatile $1.6 \pm 0.1, P=.0100$ ).

\section{Nonpulsatile Group: Comparisons Between Those With and Without Gastrointestinal Bleeding}

Finally, within the nonpulsatile device group, we compared characteristics of patients who had gastrointestinal bleeding after 15 days with those of the patients who did not have bleeding (Table 4). The only trends were toward lower BMI and longer duration of LVAD support in patients with gastrointestinal bleeding events. There were no significant differences in device purpose, diabetes status, age, sex, preoperative albumin, or bypass time between nonpulsatile device recipients with and without bleeding. Follow-up averages of heart rate, mean arterial pressure, pulse width, liver function tests, and creatinine were similar as well. The mortality was different between patients with and without bleeding with $3(25 \%)$ and $5(12 \%)$ deaths, respectively, although this difference was not statistically significant $(P=.2453)$. There was a trend toward longer implant duration in patients with gastrointestinal bleeding (no bleeding $173.9 \pm 19.5$ days vs bleeding $295.7 \pm 64.5$ days, $P=.0939$ ). There was no significant difference in INR for those with and without bleeding $(2.0 \pm 0.1$ vs 2.1 $\pm 0.2, P=.8134)$.
TABLE 3. Postimplant characteristics

\begin{tabular}{|c|c|c|c|c|c|}
\hline & \multicolumn{2}{|c|}{$\begin{array}{l}\text { Nonpulsatile } \\
\qquad(\mathrm{N}=\mathbf{5 5})\end{array}$} & \multirow{2}{*}{$\begin{array}{l}\text { Pulsatile } \\
(\mathbf{N}=46)\end{array}$} & & \multirow[b]{2}{*}{$P$ value } \\
\hline & Mean $\pm \mathrm{SE}$ & $\mathbf{n}$ & & & \\
\hline Heart rate (beats/min) & $84.2 \pm 1.6$ & 49 & $68.2 \pm 1.6$ & 42 & $<.0001$ \\
\hline $\begin{array}{l}\text { Mean arterial pressure } \\
\qquad(\mathrm{mm} \mathrm{Hg})\end{array}$ & $81.1 \pm 1.8$ & 49 & $84.8 \pm 1.9$ & 42 & .1727 \\
\hline Pulse width (mm Hg) & $32.2 \pm 1.5$ & 49 & $59.0 \pm 2.6$ & 42 & $<.0001$ \\
\hline Creatinine (mg/dL) & $1.3 \pm 0.1$ & 51 & $1.3 \pm 0.1$ & 44 & .5588 \\
\hline Sodium (mEq/L) & $140.1 \pm 0.3$ & 51 & $140.7 \pm 0.4$ & 43 & .3055 \\
\hline Albumin (g/dL) & $3.9 \pm 0.1$ & 50 & $3.9 \pm 0.1$ & 39 & .8043 \\
\hline $\begin{array}{l}\text { Alanine aminotransferase } \\
\qquad(\mathrm{U} / \mathrm{L})\end{array}$ & $38.2 \pm 6.5$ & 51 & $33.1 \pm 2.9$ & 38 & .5221 \\
\hline $\begin{array}{l}\text { Aspartate } \\
\quad \text { aminotransferase (U/L) }\end{array}$ & $50.7 \pm 4.8$ & 51 & $40.4 \pm 3.1$ & 37 & .1034 \\
\hline Total bilirubin (mg/dL) & $1.5 \pm 0.7$ & 49 & $0.8 \pm 0.1$ & 38 & .3239 \\
\hline $\begin{array}{l}\text { International normalized } \\
\text { ratio }\end{array}$ & $2.0 \pm 0.1$ & 51 & $1.6 \pm 0.1$ & 23 & .0100 \\
\hline $\begin{array}{l}\text { Activated partial } \\
\text { thromboplastin } \\
\text { time (seconds) }\end{array}$ & $42.0 \pm 1.6$ & 46 & $40.9 \pm 8.0$ & 9 & .8946 \\
\hline
\end{tabular}

\section{DISCUSSION}

LVADs are effectively prolonging life for patients with CHF. As life expectancy increases, optimizing quality of life becomes imperative. Previous trials have demonstrated equivalent survival with pulsatile and nonpulsatile ventricular assist devices. ${ }^{4,5}$ Preliminary studies evaluating the efficacy and risk profile of nonpulsatile flow devices have not specifically addressed the incidence of gastrointestinal bleeding. Letsou and colleagues ${ }^{7}$ reported 3 cases of gastrointestinal bleeding among the first 21 Jarvik 2000 nonpulsatile LVAD recipients. Hetzer and associates ${ }^{8}$ had 1 case of persistent gastrointestinal bleeding among the first 24 recipients of the Incor nonpulsatile pump (Berlin Heart GmbH, Berlin, Germany). ${ }^{8}$ Gastrointestinal bleeding was not recognized as a significant cause of postoperative morbidity in the REMATCH trial or the pulsatile FDA study protocols (Novacor; World Heart Inc, Oakland, Calif; and HeartMate XVE). ${ }^{2,9,10}$

In our series of 101 patients, the rate of gastrointestinal bleeding was significantly higher for patients who received nonpulsatile LVADs. There were 63 gastrointestinal bleeding events per 100 patient-years in nonpulsatile device recipients, versus 6.8 in the pulsatile group $(P=.0004)$. Postoperative creatinine and liver function tests were within reference ranges for both groups, indicating good end-organ function. Markers for end-organ dysfunction were similarly absent in the nonpulsatile device recipients who had gastrointestinal bleeding events. The pulsatile group was more likely to begin the device period in poorer health, with 13 patients requiring emergency Levitronix LVAD placement before placement of the pulsatile device. Nonpulsatile devices are only available to patients enrolled in one of the ongoing FDA investigational device exemption protocols. 
TABLE 4. Comparison of nonpulsatile device recipients with and without gastrointestinal bleeding events

\begin{tabular}{|c|c|c|c|}
\hline & No bleeding $(\mathrm{N}=43)$ & Bleeding $(\mathrm{N}=12)$ & $P$ value \\
\hline Age $(y$, mean $\pm S D)$ & $53.8 \pm 2.1$ & $60.1 \pm 4.5$ & .2236 \\
\hline Sex (male) & $32(74 \%)$ & $10(83 \%)$ & .5204 \\
\hline Diabetes (No.) & $14(34 \%, \mathrm{n}=41)$ & $4(40 \%, n=10)$ & .7284 \\
\hline Preoperative body mass index $\left(\mathrm{kg} / \mathrm{m}^{2}\right.$, mean $\left.\pm \mathrm{SD}\right)$ & $28.0 \pm 0.9$ & $23.9 \pm 1.8$ & .0513 \\
\hline Preoperative sodium $(\mathrm{mEq} / \mathrm{L}$, mean $\pm \mathrm{SD})$ & $136.3 \pm 1.0(\mathrm{n}=40)$ & $135.1 \pm 1.8(\mathrm{n}=10)$ & .6072 \\
\hline Preoperative albumin $(\mathrm{g} / \mathrm{dL}$, mean $\pm \mathrm{SD})$ & $3.7 \pm 0.1(\mathrm{n}=40)$ & $3.4 \pm 0.2(\mathrm{n}=10)$ & .2509 \\
\hline Etiology of heart failure (No.) & $(\mathrm{n}=42)$ & $(\mathrm{n}=12)$ & .2531 \\
\hline Acute cardiomyopathy & $1(2 \%)$ & $2(17 \%)$ & \\
\hline Chronic cardiomyopathy & $15(36 \%)$ & $2(17 \%)$ & \\
\hline Acute ischemia & $2(5 \%)$ & $1(8 \%)$ & \\
\hline Chronic ischemia & $22(52 \%)$ & $7(58 \%)$ & \\
\hline Other & $2(5 \%)$ & 0 & \\
\hline Duration of support (mo, mean $\pm \mathrm{SD}$ ) & $173.9 \pm 19.5$ & $295.7 \pm 64.5$ & .0939 \\
\hline \multicolumn{4}{|l|}{ Device purpose (No.) } \\
\hline Bridge to transplant & $37(86 \%)$ & $9(75 \%)$ & .3604 \\
\hline Destination therapy & $6(14 \%)$ & $3(25 \%)$ & .3604 \\
\hline Cardiopulmonary bypass time (min, mean $\pm \mathrm{SD}$ ) & $123.1 \pm 6.1(\mathrm{n}=43)$ & $127.6 \pm 10.6(\mathrm{n}=11)$ & .7364 \\
\hline \multicolumn{4}{|l|}{ Device type (No.) } \\
\hline HeartMate II* & $29(67 \%)$ & $9(75 \%)$ & .1539 \\
\hline MicroMed $\dagger$ & $5(12 \%)$ & $3(25 \%)$ & \\
\hline VentrAssist $\ddagger$ & $9(21 \%)$ & 0 & \\
\hline International normalized ratio (mean $\pm \mathrm{SD}$ ) & $2.0 \pm 0.1(\mathrm{n}=41)$ & $2.1 \pm 0.2(\mathrm{n}=10)$ & .8134 \\
\hline Aspartate aminotransferase $(\mathrm{U} / \mathrm{L}$, mean $\pm \mathrm{SD})$ & $53.0 \pm 5.9(\mathrm{n}=41)$ & $41.5 \pm 3.6(n=10)$ & .3525 \\
\hline Alanine aminotransferase $(\mathrm{U} / \mathrm{L}$, mean $\pm \mathrm{SD})$ & $41.2 \pm 8.0(\mathrm{n}=41)$ & $25.6 \pm 3.5(\mathrm{n}=10)$ & .3452 \\
\hline Total bilirubin $(\mathrm{mg} / \mathrm{dL}$, mean $\pm \mathrm{SD})$ & $1.7 \pm 0.9(\mathrm{n}=39)$ & $0.8 \pm 0.1(\mathrm{n}=10)$ & .6089 \\
\hline Creatinine $(\mathrm{mg} / \mathrm{dL}$, mean $\pm \mathrm{SD})$ & $1.2 \pm 0.1(\mathrm{n}=41)$ & $1.4 \pm 0.2(\mathrm{n}=10)$ & .4728 \\
\hline Mean arterial pressure $(\mathrm{mm} \mathrm{Hg}$, mean $\pm \mathrm{SD})$ & $82.5 \pm 2.0(\mathrm{n}=39)$ & $75.9 \pm 4.5(\mathrm{n}=10)$ & .2067 \\
\hline Pulse width (mean $\pm \mathrm{SD})(\mathrm{mm} \mathrm{Hg})$ & $31.5 \pm 1.9(\mathrm{n}=39)$ & $34.8 \pm 2.1(\mathrm{n}=10)$ & .4067 \\
\hline Heart rate (beats/min, mean $\pm \mathrm{SD}$ ) & $85.2 \pm 2.0(\mathrm{n}=39)$ & $80.4 \pm 2.2(\mathrm{n}=10)$ & .2375 \\
\hline Deaths (No.) & $5(12 \%)$ & $3(25 \%)$ & .2453 \\
\hline
\end{tabular}

All postoperative values represent an average from two subsequent follow-up visits 1 month apart. *Thoratec Corporation, Pleasanton, Calif. $\nmid$ MicroMed Cardiovascular, Inc, Houston, Tex. ‡๋ventracor Inc, Foster City Calif.

Therefore nonpulsatile device recipients had enough clinical stability to await completion of the 1- to 2-day informed consent and randomization process before device placement. The pulsatile group had longer bypass times $(149.0 \pm 8.1$ vs $124.1 \pm 5.3$ minutes, $P=.0115$ ), further increasing the risk for bowel ischemia and thus potentially the risk for gastrointestinal bleeding. Despite these risk factors, the pulsatile group demonstrated the lower incidence of gastrointestinal bleeding.

Among the 3 pulsatile device recipients with bleeding events, 2 events were due to ischemic bowel near the time of death. The third pulsatile device recipient with bleeding had sepsis during his gastrointestinal bleeding, with an indeterminate source despite an extensive work-up. Twelve patients in the nonpulsatile device group had bleeding events: 4 had documented arteriovenous malformations (AVMs) and 5 bled from anatomic problems, including polyps, gastric feeding tube, and mucosal erosion presumed related to gastroesophageal reflux. We were unable to identify a discrete source for bleeding in the remaining 3 patients.
The working diagnosis for 2 of these patients was small bowel AVM. The final patient was believed to have ischemic bowel.

One obvious criticism of this study is the difference in anticoagulation between the two groups. Nonpulsatile device recipients receive anticoagulation with warfarin sodium to an INR goal of 1.5 to 3. Pulsatile device recipients do not receive anticoagulation. Both nonpulsatile and pulsatile device recipients take a daily aspirin tablet. Unlike earlier case reports, in which bleeding continued until the time of transplant, all of the gastrointestinal bleeding in this series responded to an interruption in anticoagulation and lowering of the pump speed to allow greater pulsatile flow. There was no significant difference between the average INR observed at the time of bleeding in nonpulsatile device recipients and the average INR at follow-up in those without bleeding. Comparing LVAD gastrointestinal bleeding rates to bleeding complications in patients receiving anticoagulation for other reasons suggests that our findings are not due to anticoagulation alone. The gastrointestinal bleeding rate in the 
nonpulsatile group was much greater than the rate of all types of bleeding in patients receiving anticoagulation after placement of a mechanical valve. Cannegieter and coworkers ${ }^{9}$ reported a bleeding complication rate of 2.68 to 4.6 events/100 patient-years in mechanical valve recipients with combined antiplatelet and warfarin therapy. Levine and colleagues ${ }^{10}$ studied patients receiving anticoagulation for any reason and found hemorrhagic complication rates of $5.7 \%$ per year. Our finding of 63 gastrointestinal bleeding events/100 patient-years in nonpulsatile device recipients clearly exceeds that seen in patients receiving anticoagulation for other reasons. In addition, the INR range targeted for our patient population is much lower than the levels reported in these other studies.

If anticoagulation alone were the cause for our findings, we would expect to see a higher rate of bleeding from all causes in nonpulsatile device recipients. Overall bleeding rates for patients with nonpulsatile and pulsatile LVADs, however, are quite similar. The REMATCH trial had a bleeding rate at 6-month follow-up of $42 \%$ for the 68 pulsatile LVAD recipients. $^{2}$ The Texas Heart Institute follow-up of 280 HeartMate pulsatile LVAD recipients reported an all-cause bleeding rate of $48 \%{ }^{3}$ Bleeding from any cause requiring 2 units of blood in the HeartMate II LVAD (nonpulsatile) trial occurred in $53 \%$ of the 133 patients followed up for 180 days after device implantation. ${ }^{5}$ Our findings demonstrate an isolated increase in gastrointestinal bleeding for patients with nonpulsatile devices, despite similar overall bleeding rates in nonpulsatile and pulsatile LVAD recipients.

Patients with aortic stenosis (AS) demonstrate a similar isolated increase in gastrointestinal bleeding. In 1958, Heyde ${ }^{11}$ described an association between AS and gastrointestinal bleeding. Follow-up studies have found a 100-fold increase in the risk for gastrointestinal bleeding in patients with AS. ${ }^{12}$ Patients with gastrointestinal angiodysplasia who acquire AS demonstrate a decrease in high-molecular weight (HMW) multimers of von Willebrand factor (vWF). Warkentin and coworkers ${ }^{13}$ first described this acquired von Willebrand disease in 1992 as the potential link between gastrointestinal bleeding and AS. Later, Veyradier and associates ${ }^{14}$ demonstrated that patients with valvular heart disease had low levels of the largest multimers of vWF. HMW vWF multimers are believed essential for platelet-mediated hemostasis and prevention of bleeding in high-shear areas. The tortuous vessels seen in gastrointestinal angiodysplasia demonstrate high-shear stress blood flow. In AS, deformation of $\mathrm{VWF}$ as it crosses the calcific aortic valve results in a structural change, leading to proteolysis and a decrease in the number of circulating HMW multimers. ${ }^{12,15}$ After aortic valve replacement, HMW vWF multimer levels rise and gastrointestinal bleeding stops. ${ }^{15,19}$ Hypothetically, the continuous impeller mechanism of the nonpulsatile LVAD pump may result in vWF deformation, proteolysis, and ultimately deficiency of HMW vWF multimers. Patients with preexisting gastrointestinal angiodysplasia would be at risk for gastrointestinal bleeding under these conditions. Prospective evaluation of HMW vWF levels before and after nonpulsatile and pulsatile device placement is underway to test this hypothesis. Potapov and colleagues ${ }^{16}$ have demonstrated that in some settings a nonpulsatile LVAD can generate pulsatile flow as a result of improved contractility of the recovering left ventricle. Decreasing nonpulsatile device flow could result in pulsatile blood flow and potentially decrease vWF deformation and proteolysis. Restoration of HMW levels in this way could prevent or resolve gastrointestinal bleeding.

Nonpulsatile flow may actually increase the development of gastrointestinal angiodysplasia. Investigators have proposed that the narrow pulse pressure that occurs in AS and in nonpulsatile device recipients may increase intraluminal pressure and dilate mucosal veins, leading to AVM pathogenesis. ${ }^{7}$ These AVMs are believed to be more vulnerable to bleeding during stress and anticoagulation. ${ }^{7,14}$ Cappell and Lebwohl ${ }^{17}$ have suggested that narrow pulse pressure triggers an increase in sympathetic tone, causing smooth muscle relaxation, arteriovenular dilatation, and ultimately arteriovenous malformation. Saito and colleagues ${ }^{18}$ examined sheep implanted with nonpulsatile LVADs and demonstrated thinning of the medial layer of the ascending aorta. Whether there are such changes throughout the arterial vascular system in human beings is unknown.

\section{CONCLUSIONS}

We have documented an increased rate of gastrointestinal bleeding in patients implanted with nonpulsatile LVADs relative to that seen with pulsatile flow devices. The bleeding rate was higher than would be expected from anticoagulation alone. Propensity toward arteriovenous malformation in combination with low levels of hemostasis-enhancing HMW von Willebrand factor multimers provides casual mechanism. Further prospective investigation is required to confirm these findings and to identify risk factors for gastrointestinal bleeding in this population.

\section{References}

1. American Heart Association web site: Heart Failure, last updated 12/06/07. URL: www.americanheart.org/presenter.jhtml?identifier $=1486$.

2. Rose EA, Gelijns AC, Moskowitz AJ, Heitjan DF, Stevenson LW, Dembitsky W, et al. Long-term use of a left ventricular assist device for end-stage heart failure. N Engl J Med. 2001;345:1435-43.

3. Frazier DH, Rose EA, Mehmet CO, Dembitsky W, McCarthy P, Radovancevic B, et al. Multicenter clinical evaluation of the HeartMate vented electric left ventricular assist system in patients awaiting heart transplantation. J Thorac Cardiovasc Surg. 2001;122:1186-95.

4. Feller ED, Sorensen EN, Haddad M, Pierson RN, Johnson FL, Brown JM, et al Clinical outcomes are similar in pulsatile and non-pulsatile left ventricular assist device recipients. Ann Thorac Surg. 2007;83:1082-8.

5. Miller LW, Pagani FD, Russell SD, John R, Boyle AJ, Aaronson KD, et al. Use of a continuous-flow device in patients awaiting heart transplantation. $N$ Engl J Med 2007;357:885-96.

6. Letsou GV, Myers TJ, Gregoric ID, Delgado R, Shah N, Robertson K, et al. Continuous axial-flow left ventricular assist device (Jarvik 2000) maintains 
kidney and liver perfusion for up to 6 months. Ann Thorac Surg. 2003;76: 1167-70.

7. Letsou GV, Shah N, Gregoric ID, Myers TJ, Delgado R, Frazier OH. Gastrointestinal bleeding from arteriovenous malformations in patients supported by the Jarvik 2000 axial-flow left ventricular assist device. J Heart Lung Transplant. 2005; 24:105-9.

8. Hetzer R, Weng Y, Potapov EV, Pasic M, Drews T, Jurmann M, et al. First experiences with a novel magnetically suspended axial flow left ventricular assist device. Eur J Cardiothorac Surg. 2004;25:964-70.

9. Cannegieter SC, Rosendaal FR, Briet E. Thromboembolic and bleeding complications in patients with mechanical heart valve prostheses. Circulation. 1994;89: 635-41.

10. Levine MN, Raskob G, Beyth RJ, Kearon C, Schulman S. Hemorrhagic complications of anticoagulant treatment: the seventh ACCP conference on antithrombotic and thrombolytic therapy. Chest. 2004;126:287-310.

11. Heyde EC. Gastrointestinal bleeding in aortic stenosis [letter]. $N$ Engl J Med. 1958;259:196.

12. Warkentin TE, Moore JC, Anand SS, Lonn EM, Morgan DG. Gastrointestinal bleeding, angiodysplasia, cardiovascular disease, and acquired von Willebrand syndrome. Transfus Med Rev. 2003;17:272-86.
13. Warkentin TE, Moore JC, Morgan DG. Aortic stenosis and bleeding gastrointestinal angiodysplasia: is acquired von Willebrand's disease the link? Lancet. 1992;340:35-7.

14. Veyradier A, Balian A, Wolf M, Giraud V, Montembault S, Obert B, et al. Abnormal von Willebrand factor in bleeding angiodysplasias of the digestive tract. Gastroenterology. 2001;120:346-53.

15. Vincentelli A, Susen S, Le Tourneau T, Six I, Fabre O, Juthier F, et al. Acquired von Willebrand syndrome in aortic stenosis. $N$ Engl J Med. 2003;349: 343-9.

16. Potapov EV, Loebe M, Nasseri BA, Sinawski H, Koster A, Kuppe H, et al. Pulsatile flow in patients with a novel nonpulsatile implantable ventricular assist device. Circulation. 2000;102:183-7.

17. Cappell MS, Lebwohl O. Cessation of recurrent bleeding from gastrointestinal angiodysplasias after aortic valve replacement. Ann Intern Med. 1986;105: 55-7.

18. Saito S, Westaby S, Piggot D, Dudnikov S, Robson D, Catarino PA, et al. Endorgan function during chronic nonpulsatile circulation. Ann Thorac Surg. 2002; 74:1080-5.

19. Yoshida K, Tobe S, Kawata M, Yamaguchi M. Acquired and reversible von Willebrand disease with high shear stress aortic valve stenosis. Ann Thorac Surg. 2006;81:490-4.

\section{Appendix. Nonpulsatile investigational device exemption protocol example inclusion and exclusion criteria}

\section{Inclusion Criteria}

1. Patient or legal representative has signed an informed consent form.

2. Patient is listed for transplant.

3. Body surface area is at least $1.2 \mathrm{~m}^{2}$.

4. New York Heart Association functional class IV heart failure symptoms are present.

5. Female patients with childbearing potential must agree to use adequate contraceptive precautions (defined as oral contraceptives, intrauterine devices, surgical contraception, or a combination of condom and spermicide) for the duration of the study.

6. Patient is receiving inotropic support, if tolerated.

7. Despite medical therapy, the patient must meet one of the following criteria:

a. no contraindication for listing as status $1 \mathrm{~A}$ or

b. no contraindication for listing as status $1 \mathrm{~B}$ and meeting the following hemodynamic criteria (collected within 48 hours of enrollment):

i. pulmonary capillary wedge pressure or pulmonary artery diastolic pressure (PAD) of at least $20 \mathrm{~mm}$ $\mathrm{Hg}$ and

ii. cardiac index no greater than $2.2 \mathrm{~L} /\left(\mathrm{min} \cdot \mathrm{m}^{2}\right)$ or systolic blood pressure no greater than $90 \mathrm{~mm} \mathrm{Hg}$.

\section{Exclusion Criteria}

1. Etiology of heart failure is due to or associated with uncorrected thyroid disease, obstructive cardiomyopathy, pericardial disease, amyloidosis, or restrictive cardiomyopathy.

2. Technical obstacles pose an inordinately high surgical risk in the judgment of the investigator.
3. Any ongoing mechanical circulatory support other than intra-aortic balloon counterpulsation is present.

4. BMI is $40 \mathrm{~kg} / \mathrm{m}^{2}$ or greater.

5. Pregnancy test result is positive in woman of childbearing potential.

6. Mechanical aortic cardiac valve is present and will not be converted to a bioprosthesis at the time of LVAD implant.

7. Patient has a history of cardiac transplant.

8. Platelet count is no higher than 50,000 cells $/ \mathrm{mL}$.

9. There is evidence of an untreated aortic aneurysm at least $5 \mathrm{~cm}$ in size.

10. Psychiatric disease, irreversible cognitive dysfunction, or psychosocial issues are likely to impair compliance with the study protocol and LVAD management.

11. An active, uncontrolled infection is present.

12. Patient has intolerance to anticoagulant or antiplatelet therapies or any other perioperative or postoperative therapy the investigator may mandate according to the patient's health status.

13. Any one of the following risk factors for and indicators of severe end-organ dysfunction or failure is present:

a. INR of at least 2.5 not caused by anticoagulant therapy of clopidogrel bisulfate (INN clopidogrel) administration within 5 days;

b. total bilirubin greater than $5 \mathrm{mg} / \mathrm{dL}$, or shock liver (eg, transaminases greater than $2000 \mathrm{U} /$ liter), or biopsyproven liver cirrhosis;

c. history of severe chronic obstructive pulmonary disease or severe restrictive lung disease;

d. fixed pulmonary hypertension, with a most recent peripheral vascular resistance greater than 6 Wood units, that is unresponsive to pharmacologic intervention; 
e. history of unresolved stroke or uncorrectable cerebrovascular disease;

f. serum creatinine of at least $3.5 \mathrm{mg} / \mathrm{dL}$ or the need for longterm renal replacement therapy (eg, long-term dialysis);

g. significant peripheral vascular disease accompanied by rest pain or extremity ulceration.
14. The patient has moderate to severe aortic insufficiency without plans for correction during pump implantation surgery.

15. The patient is participating in any other clinical investigation that is likely to confound study results or affect study outcome. 